\title{
ĐÁNH GIÁ Độ CHÍNH XÁC CỦA MÔ HÌNH QUASIGEOID QUỐC GIA KHỞI ĐÂU VIGAC2014 DỰA TRÊN CƠ SỞ DỮ LIỆU CỦA 75 ĐIỂM ĐỘ CAO HẠNG II
}

\author{
LƯO'NG THANH THACH \\ Trường Đại học Tài nguyên và Môi trường Hà Nội
}

\section{Tóm tắt:}

Bài báo khoa học này tiến hành đánh giá kiểm tra giá trị thế trọng trường $W_{0}$ $=62636847.291 \mathrm{~m}^{2} . \mathrm{s}^{-2}$ và giá trị độ cao $\mathrm{H}_{0}=0.890 \mathrm{~m}$ của mặt quasigeoid cục bộ Hòn Dấu, mà bản chất là đánh giá độ chính xác của mô hình quasigeoid quốc gia khởi đầu VIGAC2014 dựa trên cơ sở dữ liệu của 75 điểm độ cao hạng II nhà nước. Các kết quả kiểm tra tiếp tục khẳng định sự tin cậy của các giá trị nêu trên làm cơ sở khoa học để giải quyết các bài toán trắc địa vật lý hiện đại ở nước ta.

\section{1. Đặt vấn đề}

Năm 1975, lần đầu tiên trên thế giới nhà trắc địa người Séc Bursa Milan đã xác định được thế trọng trường của mặt geoid toàn cầu từ số liệu đo cao vệ tinh (altimetry). Từ đó đến nay, dựa trên giá trị thế trọng trường của mặt geoid toàn cầu các tổ chức quốc tế, các nước hoặc nhóm nước đã nghiên cứu xác định và công bố các giá trị thế trọng trường của mặt geoid/quasigeoid cục bộ dựa trên các số liệu đo cao vệ tinh, số liệu trọng lực, số liệu GNSS/Thủy chuẩn... kết hợp với mô hình trọng trường Trái Đất toàn cầu EGM2008 hoặc các mô hình trọng trường Trái Đất toàn cầu với giá trị thế trọng trường xác định.

Giá trị thế trọng trường $\bar{W}_{0}=62636856.0 \mathrm{~m}^{2} \cdot \mathrm{s}^{-2}$ của mặt geoid toàn cầu trên các biển và các đại dương thế giới được xác định nhờ các dữ liệu altimetry và được công bố trong tài liệu (Bursa M., Kenyon S., Kouba J., Radj K., Vatrt V., Vojtiskov., Simek J. (2002)). Giá trị thế trọng trường này đã được Tổ chức dịch vụ quay Trái Đất quốc tế IERS (International Earth Rotation Service) công nhận trong các Hiệp ước (Conventions) năm 2004 và 2010 (Dennis D. McCarthy and Gerard Petit (2004), Petit G., Luzum B.(2010). IERS Conventions (2010)) và được dùng để xây dựng mô hình trọng trường Trái Đất toàn cầu EGM2008.

Theo công bố trong tài liệu (Nadim DAYOUB (Syria), Philip MOORE, Stuart J. EDWARDS and Nigel T. PENNA (The United Kingdom) (2011)), các nước vùng Baltic đã sử dụng các số liệu TGO - đo mực nước ở các trạm nghiệm triều, GPS (Global Positioning System), Thủy chuẩn kết hợp với việc sử dụng các hệ số điều hòa cầu của mô hình trọng trường Trái Đất toàn cầu EGM96 đã xác định được giá trị thế trọng trường $W_{0}=$ $62636855.75 \pm 0.21 \mathrm{~m}^{2} . \mathrm{s}^{-2}$.

Theo tài liệu (Tenzer R., Vatrt V., Amos M (2009)), mô hình trọng trường toàn cầu EGM2008 và các dữ liệu GPS/Thủy chuẩn được sử dụng để xác định sự khác biệt giữa mô hình quasigeoid khu vực NZGeoid05 (New Zealand Geoid of 2005) và mô hình quasigeoid toàn cầu EGM2008 cho giá trị độ chênh là $0.56 \mathrm{~m}$. Các phân tích tương tự được thực hiện cho mô hình NZGeoid2009 (New Zealand Geoid of 2009) - là mô hình quasigeoid 
quốc gia chính thức của New Zealand, kết quả độ chênh giữa NZGeoid2009 so với mô hình quasigeoid toàn cầu EGM2008 là $0.51 \mathrm{~m}$.

Theo phân tích trong tài liệu (Hà Minh Hòa (2012b)), trên thế giới hiện có 2 phương pháp để xác định giá trị thế trọng trường $W_{0}$ của mặt geoid cục bộ sát nhất với mặt biển trung bình cục bộ:

Phương pháp thứ nhất là sử dụng các mô hình MDT (Mean Dynamic Topography) và kết quả đo GPS tại trạm nghiệm triều 0 (trạm nghiệm triều mà mặt nước biển trung bình tại đó được chọn là mặt khởi tính cho hệ độ cao quốc gia). Theo phương pháp này, giá trị thế trọng trường thực $W_{0}$ được xác định theo công thức:

$$
W_{0}=\bar{W}_{0}-\bar{\gamma}_{0}(M D T+T G O)
$$

Ở đây $\bar{W}_{0}$ - thế trọng trường thực của geoid toàn cầu được sử dụng trong mô hình $\mathrm{MDT}$, $\bar{F}_{t}$ - gia tốc lực trọng trường chuẩn của mặt ellipsoid, TGO - độ lệch của mặt biển trung bình cục bộ so với mặt biển trung bình toàn cầu tại trạm nghiệm triều 0 do sự tồn tại của sai số hệ thống trong mô hình $\mathrm{MDT}$ toàn cầu. Phương pháp này được các nước Bắc $\mathrm{Mỹ}$, Canada, Australia và một số nước ở châu Âu sử dụng.

Phương pháp thứ hai là sử dụng các mô hình mặt biển trung bình MSS được xác định từ các dự án vệ tinh để xác định các bán kính - vectơ của các điểm chạy trên mặt biển trung bình và sử dụng các mô hình trọng trường toàn cầu $E G M$ để xác định thế trọng trường thực $W_{0}$ theo công thức sau:

$$
W(\rho, \theta, L)=\frac{G M}{\rho} \cdot\left\{1+\sum_{n=2}^{\infty}\left(\frac{a_{e}}{\rho}\right)^{n}\left[\sum_{k=0}^{n}\left(C_{n k} \cdot \cos (k L)+S_{n k} \cdot \sin (k \lambda)\right) \cdot P_{n k}(\theta)\right]\right\}+\frac{\omega^{2}}{2} \cdot \rho^{2} \cdot \sin ^{2} \theta
$$

ở đây $\theta=90^{\circ}-B$. Phương pháp này được các nước Nam Mỹ, Vương quốc Anh, Pháp và Đức sử dụng.

Tuy nhiên, ở Việt Nam, các mốc độ cao quốc gia đã được xây dựng qua nhiều thập kỷ dẫn đến nhiều mốc đã bị chuyển dịch do các tác động nhân sinh và tự nhiên mà không được duy tu bảo trì, nên việc sử dụng các điểm độ cao hạng I, II vào mục đích nghiên cứu khoa học sẽ làm sai lệch các kết quả nghiên cứu. Để khắc phục vấn đề này, đồng thời tận dụng được các điểm độ cao hạng I ổn định để xác định giá trị thế trọng trường thực $W_{0}$ của mặt geoid cục bộ Hòn Dấu, tác giả Hà Minh Hòa trong tài liệu (Hà Minh Hòa (2012a)) đã đề xuất phương pháp thứ ba để xác định thế trọng trường thực $W_{0}$ của mặt geoid cục bộ Hòn Dấu kết hợp với việc giải quyết bài toán xây dựng mô hình quasigeoid quốc gia dựa trên phương trình tương đương của các dị thường độ cao GPS/thủychuẩn và dị thường độ cao trọng lực kết hợp với các điểm độ cao hạng I quốc gia được đo và xử lý dữ liệu GPS trong ITRF. Theo đó, dựa trên 35 điểm độ cao hạng I được phân bố đồng đều trên lãnh thổ Việt Nam, trong các tài liệu (Hà Minh Hòa (2012b); Hà Minh Hòa (2012c); Hà Minh Hòa (2014)), đã xác định được giá trị thế trọng trường thực $W_{0}=(62636847.291$ 1 0.183) $\mathrm{m}^{2} \cdot \mathrm{s}^{-2}$ của mặt geoid cục bộ sát nhất với mặt biển trung bình nhiều năm tại trạm nghiệm triều Hòn Dấu.

Để kiểm tra giá trị thế trọng trường thực $W_{0}$ của mặt geoid cục bộ Hòn Dấu, trong tài 
liệu (Hà Minh Hòa, Nguyễn Thị Thanh Hương, Lương Thanh Thạch (2015b)) đã sử dụng phương pháp thứ nhất và cho độ chênh kết quả xác định theo hai phương pháp chỉ ở mức $0.005 \mathrm{~m}^{2} \cdot \mathrm{s}^{-2}$ tương ứng với độ xê dịch của các mặt đẳng thế cục bộ tại trạm nghiệm triều Hòn Dấu ở mức nhỏ bỏ qua là $0.5 \mathrm{~mm}$. Điều này một lần nữa xác định tính đúng đắn của phương pháp thứ ba.

Trong các tài liệu (Hà Minh Hòa (2012b);Hà Minh Hòa (2014)) đã sử dụng 11 điểm trọng lực cơ sở và 29 điểm trọng lực hạng I phủ trùm cả nước để xác định độ cao của mặt geoid cục bộ Hòn Dấu so với mặt geoid toàn cầu và nhận được giá trị $H_{0}=0.890 \mathrm{~m}$ và là đại lượng không đổi trên toàn lãnh thổ Việt Nam. Kết quả này cũng được khẳng định một lần nữa trong tài liệu (Nguyễn Tuấn Anh (2015)) khi sử dụng 133 điểm trọng lực chi tiết được phân bố đều trên 09 vùng lãnh thổ Việt Nam: Tây Bắc, Đông Bắc, Tây Tây Bắc, Đông Đông Bắc, Bắc Trung Bộ, Trung Bộ, Nam Trung Bộ, Tây Nguyên và Nam Bộ.

Do tính chất quan trọng của giá trị thế trọng trường $W_{0}=(62636847.291 \pm 0.183) \mathrm{m}^{2} \cdot \mathrm{s}^{-2}$ của mặt geoid cục bộ Hòn Dấu và sự không đổi về độ cao của mặt geoid cục bộ Hòn Dấu so với mặt geoid toàn cầu (bằng $0.890 \mathrm{~m}$ ) trong việc giải quyết các bài toán vật lý hiện đại ở nước ta, bài báo khoa học này sẽ kiểm tra, đánh giá hai giá trị nêu trên bằng dữ liệu của 75 điểm độ cao hạng II quốc gia và 09 điểm cơ sở hạng I của mô hình VIGAC2014, mà thực chất là đi đánh giá độ chính xác của mô hình VIGAC2014.

\section{Giải quyết vấn đề}

Mạng lưới độ cao quốc gia được hoàn thiện, bổ sung bằng phương pháp thủy chuẩn truyền thống cách đây khoảng 40 năm $(1976$ - 1987). Theo kết quả công bố trong tài liệu (Vũ Xuân Cường (2015)), năm 2011 đã tiến hành đo kiểm tra một số mốc độ cao hạng I quốc gia, kết quả kiểm tra cho thấy độ chênh với giá trị độ cao được Cục Đo đạc và Bản đồ Việt Nam cung cấp dao động trong khoảng giá trị $0.2 \mathrm{~m}$ đến $0.6 \mathrm{~m}$.

Công tác xử lý số liệu trắc địa là tìm kiếm, phát hiện và loại bỏ trị đo có sai số thô. Đối với tập hợp dữ liệu các điểm độ cao hạng II, dùng tiêu chuẩn Smirnov và điều kiện sai số giới hạn không lớn hơn 2.0 đến 2.5 lần sai số trung phương, kết quả lựa chọn được 75 điểm độ cao hạng II đủ độ tin cậy để tiến hành đánh giá mô hình VIGAC2014.

Trong hàng loạt các công trình, ví dụ như (Hà Minh Hòa, Nguyễn Thị Thanh Hương, Lương Thanh Thạch (2015b); Hà Minh Hòa và nnk (2016); Nguyễn Tuấn Anh (2015)) đã thực hiện đánh giá mô hình VIGAC2014 dựa trên 89 điểm hạng I, 133 điểm trọng lực chi tiết được phân bố trên 09 vùng lãnh thổ Việt Nam và 26 điểm thuộc mạng lưới trắc địa địa động lực. Kết quả đều cho thấy mô hình VIGAC2014 có thể dùng để xác định độ cao chuẩn có độ chính xác tương đương hạng II trên lãnh thổ Việt Nam. Để có thêm cơ sở nhằm đưa mô hình VIGAC2014 vào ứng dụng trong thực tế, trong bài báo khoa học này sẽ tiếp tục đánh giá mô hình VIGAC2014 dựa trên 75 điểm độ cao hạng II. Kết quả của các bước tính toán được trình bày trong bảng 1 dưới đây. Giá trị trong cột (8) ở bảng 1 là dị thường độ cao toàn cầu được xác định từ mô hình EGM2008 và được chuyển về hệ triều 0 . Quá trình tính toán các giá trị trong các cột(4),(5), (7), (8), (9), (10) của bảng 1 đã được trình bày trong các tài liệu (Hà Minh Hòa (2014), Hà Minh Hòa và nnk (2016))và được tóm tắt lại dưới dạng các công thức sau:

* Chuyển đổi độ cao trắc địa giữa các hệ triều của điểm M: 


$$
\left(H_{M}\right)_{z}=\left(H_{M}\right)_{m}=\left(H_{M}\right)_{n}+h_{2} \cdot\left(0.099-0.296 \cdot \sin ^{2} B\right)<m>
$$

ở đây, $\left(H_{M}\right)_{n}$ là độ cao trắc địa của điểm $M$ trong hệ không phụ thuộc triều, $\left(H_{M}\right)_{m}$ là độ cao trắc địa của điểm $M$ trong hệ triều trung bình và $\left(H_{M}\right)_{z}$ là độ cao trắc địa của điểm $M$ trong hệ triều 0 , hệ số Love $h_{2}=0.627, B$ là vĩ độ trắc địa của điểm $\mathrm{M}$.

* Chuyển dị thường độ cao từ hệ không phụ thuộc triều về hệ triều 0 của điểm $\mathrm{M}$ :

$$
\left(\zeta_{M}\right)_{z}=\left(\zeta_{M}\right)_{n}+k_{2} \cdot \Delta \rho_{0}=\left(\zeta_{M}\right)_{n}+k_{2} \cdot\left(0.099-0.296 \cdot \sin ^{2} B\right)<m>
$$

ở đây, $\left(\zeta_{M}\right)_{n}$ là dị thường độ cao của điểm $M$ trong hệ không phụ thuộc triều, $\left(\zeta_{M}\right)_{z}$ là dị thường độ cao của điểm $M$ trong hệ triều 0 , hệ số Love $k_{2}=0.29$.

* Chuyển đổi độ cao chuẩn giữa các hệ triều

$$
\left(H_{M}^{\gamma}\right)_{z}=\left(H_{M}^{\gamma}\right)_{m}+\Delta \rho_{0}=\left(H_{M}^{\gamma}\right)_{m}+0.099-0.296 \cdot \sin ^{2} B .<m>
$$

ở đây $\left(H_{M}^{\gamma}\right)_{z}$ là độ cao chuẩn của điểm $M$ trong hệ triều $0,\left(H_{M}^{\gamma}\right)_{m}$ là độ cao chuẩn của điểm $M$ trong hệ triều trung bình và $\left(H_{M}^{\gamma}\right)_{n}$ là độ cao chuẩn của điểm $M$ trong hệ không phụ thuộc triều.

* Xác định độ cao chuẩn bằng 09 điểm cơ sở hạng I:

Trong tài liệu (Hà Minh Hòa và nnk (2016)) đã xác định được 09 điểm cơ sở hạng I của mô hình VIGAC2014 được trình bày ở bảng 2 . Để xem xét tính đồng đều về độ chính xác của mô hình VIGAC2014, dựa trên 09 điểm cơ sở này, tiến hành kiểm tra độc lập xác suất độ cao chuẩn của 06 điểm hạng II phân bố đều trên lãnh thổ Việt Nam theo các vĩ độ trắc địa $\left(9^{\circ}, 11^{\circ}, 13^{\circ}, 15^{\circ}, 17^{\circ}, 21^{\circ}\right.$ - không có điểm nào trong tập số liệu 75 điểm nằm ở vĩ độ $\left.19^{\circ}\right)$. Độ cao chuẩn của 06 điểm hạng II này được xác định từ 09 điểm cơ sở hạng I theo công thức sau:

ở đây

$$
\left(\widetilde{H}_{j}^{\gamma}\right)_{z}=\frac{\sum_{i=1}^{n}\left(H_{j}^{\gamma}\right)_{i}}{n},
$$

$$
\left(H_{j}^{\gamma}\right)_{i}=\left(H_{i}^{\gamma}\right)_{z}+\left\{\left(\bar{H}_{j}\right)_{z}-\left(\bar{H}_{i}\right)_{z}\right\}-\left\{\left(\hat{\bar{\zeta}}_{j}\right)_{z}-\left(\hat{\bar{\zeta}}_{i}\right)_{z}\right\} \text {. }
$$

Kết quả xác định độ cao chuẩn của các điểm hạng II độc lập từ 09 điểm cơ sở hạng I, đồng thời so sánh giá trị này với giá trị được xác định từ mô hình VIGAC2014 được trình bày trong các bảng 3.1 đến bảng 3.6. (Xem bảng 1)

Nhận xét: Trong tài liệu (Hà Minh Hòa (2003); Hà Minh Hòa (2014)) đã nghiên cứu xác định độ chính xác cho phép của độ cao chuẩn các hạng, theo đó, sai số trung phương lớn nhất (giới hạn) của điểm độ cao hạng II có giá trị $\left(m_{I I}\right)_{g h}= \pm 0.079(\mathrm{~m})$. So sánh kết quả đánh giá trong bảng 1 với giá trị của $\left(m_{\| l}\right)_{g h}$, ta thấy rằng giá trị độ chênh của sai số trung phương chỉ ở mức $1.7 \mathrm{~mm}$ - đây là độ chênh nhỏ bỏ qua. (Xem bảng 2, 3) 
Bảng 1: Kết quả tính độ cao chuẩn của 75 điểm độ cao hạng II theo mô hình VIGAC2014 và độ lệch so với giá trị độ cao chuẩn gốc

\begin{tabular}{|c|c|c|c|c|c|c|c|c|c|}
\hline STT & Tên điểm & $\begin{array}{c}\text { Vĩ độ trắc địa } \\
\text { B } \\
(0)\end{array}$ & $\begin{array}{c}\text { Độ cao } \\
\text { trắc địa } \\
\text { trong hệ } \\
\text { triều 0 } \\
(\mathrm{m}) \\
\left(H_{i}\right)_{z}\end{array}$ & $\begin{array}{c}\text { Độ cao } \\
\text { chuẩn } \\
\text { quốc gia } \\
\text { trong hệ } \\
\text { triều } \\
\text { trung } \\
\text { bình do } \\
\text { cục } \\
\text { ĐĐBĐ } \\
\text { VN cung } \\
\text { cấp (m) } \\
\\
\left(H_{i}^{\gamma}\right)_{m}\end{array}$ & \begin{tabular}{|c} 
Dị \\
thường \\
độ cao \\
toàn \\
cầu \\
được \\
xác định \\
từ mô \\
hình \\
EGM20 \\
08 trong \\
hệ triều \\
$0(\mathrm{~m})$ \\
$(\bar{\zeta})_{Z}$
\end{tabular} & $\begin{array}{c}\text { Dị thường độ } \\
\text { cao cục bộ được } \\
\text { xác định từ mô } \\
\text { hình VIGAC2014 } \\
\text { tương ứng với } \\
\text { mặt geoid cục } \\
\text { bộ trong hệ triều } \\
0(\mathrm{~m}) \\
\left(\zeta_{1}\right)_{z}=\left(\bar{\zeta}_{1}\right)_{2}+0.890\end{array}$ & $\begin{array}{c}\text { Độ cao chuẩn } \\
\text { được tính từ } \\
\text { mô hình } \\
\text { VIGAC2014 } \\
\text { trong hệ triều } 0 \\
(\mathrm{~m}) \\
\hat{H}_{i}^{\gamma}=\left(H_{i}\right)_{l}-\left(\zeta_{i}\right)_{z}\end{array}$ & $\begin{array}{c}\text { Độ cao } \\
\text { chuẩn } \\
\text { quốc gia } \\
\text { trong hệ } \\
\text { triều } 0 \\
(\mathrm{~m}) \\
\\
\left(H_{i}^{\gamma}\right)_{z}\end{array}$ & $\begin{array}{r}\text { Độ chênh }(\mathrm{m}) \\
\Delta_{i}=\hat{\bar{H}}_{i}^{\gamma}-\left(H_{i}^{\gamma}\right)\end{array}$ \\
\hline$(1)$ & (2) & (3) & (4) & (5) & (6) & (7) & (8) & (9) & (10) \\
\hline 1 & II(DK-TM)41 & 15.84309167 & 341.730 & 351.090 & -10.325 & -9.435 & 351.165 & 351.167 & -0.002 \\
\hline 2 & II(BH-XL)17 & 10.56814444 & 37.555 & 37.952 & -1.356 & -0.466 & 38.021 & 38.041 & -0.020 \\
\hline 3 & II (BS-CD)3 & 10.38336111 & -7.147 & 2.469 & -10.574 & -9.684 & 2.537 & 2.559 & -0.022 \\
\hline 4 & II(CT-GD)10 & 11.37010000 & 20.000 & 25.934 & -6.889 & -5.999 & 25.999 & 26.021 & -0.022 \\
\hline 5 & II(NB-HN)15 & 22.33222778 & 229.844 & 257.591 & -28.73 & -27.840 & 257.684 & 257.647 & 0.037 \\
\hline 6 & II(PLK-PL)2 & 13.99366667 & 736.799 & 739.401 & -3.611 & -2.721 & 739.520 & 739.482 & 0.038 \\
\hline 7 & II(HN-MT)5 & 10.64587778 & -4.995 & 3.130 & -9.062 & -8.172 & 3.177 & 3.219 & -0.042 \\
\hline 8 & II(CD-VC)4-1 & 10.55802500 & -5.842 & 2.812 & -9.59 & -8.700 & 2.858 & 2.901 & -0.043 \\
\hline 9 & II(LC-TG)19A & 21.42061389 & 462.119 & 494.141 & -32.928 & -32.038 & 494.157 & 494.200 & -0.043 \\
\hline 10 & II(NB-HN)27-1 & 21.96829167 & 46.011 & 74.027 & -29.007 & -28.117 & 74.128 & 74.084 & 0.044 \\
\hline 11 & II(BS-CD)7-1 & 10.44597500 & -5.672 & 3.888 & -10.584 & -9.694 & 4.022 & 3.977 & 0.045 \\
\hline 12 & II(LC-TG)15 & 21.55003333 & 429.910 & 461.892 & -32.98 & -32.090 & 462.000 & 461.951 & 0.049 \\
\hline 13 & II(MT-TH)4 & 13.58203333 & 344.039 & 345.939 & -2.823 & -1.933 & 345.972 & 346.021 & -0.049 \\
\hline 14 & II(LC-TG)31 & 21.55060556 & 488.412 & 519.692 & -32.279 & -31.389 & 519.801 & 519.751 & 0.050 \\
\hline 15 & II(NK-PT)10 & 22.05013889 & 22.291 & 51.723 & -30.431 & -29.541 & 51.832 & 51.780 & 0.052 \\
\hline 16 & II(PLK-PL)12 & 13.96926111 & 434.114 & 435.468 & -2.268 & -1.378 & 435.492 & 435.549 & -0.057 \\
\hline 17 & II(CT-GD)15-1 & 11.26700556 & -2.841 & 3.338 & -7.098 & -6.208 & 3.367 & 3.426 & -0.059 \\
\hline 18 & II(GD-AB)9-1 & 10.78073056 & -2.876 & 1.591 & -5.387 & -4.497 & 1.621 & 1.680 & -0.059 \\
\hline 19 & II(GD-APD)6-1 & 10.99192222 & 3.152 & 7.298 & -5.065 & -4.175 & 7.327 & 7.386 & -0.059 \\
\hline 20 & II(PLK-PL)8 & 14.02744722 & 714.448 & 715.969 & -2.433 & -1.543 & 715.991 & 716.051 & -0.060 \\
\hline 21 & II(CT-VT)1 & 9.913394444 & -3.597 & 1.368 & -5.883 & -4.993 & 1.396 & 1.458 & -0.062 \\
\hline 22 & $\mathrm{II}(\mathrm{CD}-\mathrm{HN}) 6$ & 10.82110556 & -4.284 & 4.700 & -10.025 & -9.135 & 4.851 & 4.789 & 0.062 \\
\hline 23 & II(SC-VT)3-1 & 9.78365000 & -4.873 & 0.743 & -6.534 & -5.644 & 0.771 & 0.834 & -0.063 \\
\hline 24 & II(NB-HN)11-1 & 22.45877778 & 211.631 & 239.492 & -28.872 & -27.982 & 239.613 & 239.547 & 0.066 \\
\hline 25 & II(GD-APD)2-1 & 11.07232778 & 2.687 & 7.577 & -5.935 & -5.045 & 7.732 & 7.665 & 0.067 \\
\hline 26 & II(BH-XL)11-1 & 10.52271111 & 1.009 & 1.950 & -1.852 & -0.962 & 1.971 & 2.039 & -0.068 \\
\hline 27 & II(BS-CD)12 & 10.56183889 & -5.250 & 4.874 & -11.033 & -10.143 & 4.893 & 4.963 & -0.070 \\
\hline
\end{tabular}


Nghiên cúu

\begin{tabular}{|c|c|c|c|c|c|c|c|c|c|}
\hline 28 & II(TX-TL)6 & 10.63025556 & -1.355 & 1.319 & -3.581 & -2.691 & 1.336 & 1.407 & -0.071 \\
\hline 29 & II(TX-TL)25 & 10.35857222 & -1.189 & 1.985 & -4.08 & -3.190 & 2.001 & 2.074 & -0.073 \\
\hline 30 & $\mathrm{II}(\mathrm{BH}-\mathrm{XL}) 6$ & 10.77301111 & 4.613 & 6.120 & -2.559 & -1.669 & 6.282 & 6.208 & 0.074 \\
\hline 31 & $\mathrm{II}(\mathrm{YB}-\mathrm{CN}) 18$ & 21.20455000 & 159.920 & 187.883 & -28.835 & -27.945 & 187.865 & 187.943 & -0.078 \\
\hline 32 & II(NB-HN)24 & 22.07952500 & 71.851 & 99.636 & -28.811 & -27.921 & 99.772 & 99.693 & 0.079 \\
\hline 33 & $\mathrm{II}(\mathrm{MC}-\mathrm{XM}) 7-1$ & 20.69791667 & 164.832 & 191.545 & -27.586 & -26.696 & 191.528 & 191.607 & -0.079 \\
\hline 34 & II(GD-AB)12 & 10.66122500 & -3.583 & 1.005 & -5.487 & -4.597 & 1.014 & 1.094 & -0.080 \\
\hline 35 & II $(\mathrm{HN}-\mathrm{AB}) 3$ & 10.81671111 & -4.755 & 3.507 & -9.155 & -8.265 & 3.510 & 3.595 & -0.085 \\
\hline 36 & $\mathrm{II}(\mathrm{HN}-\mathrm{AB}) 11$ & 10.85062222 & -4.143 & 2.867 & -7.901 & -7.011 & 2.868 & 2.955 & -0.087 \\
\hline 37 & II(PLK-PL)16 & 13.95565278 & 414.435 & 415.534 & -1.981 & -1.091 & 415.526 & 415.616 & -0.090 \\
\hline 38 & II(GD-AB)3-1 & 10.95284722 & -3.833 & 1.334 & -6.053 & -5.163 & 1.330 & 1.422 & -0.092 \\
\hline 39 & II $(\mathrm{HN}-\mathrm{AB}) 23$ & 10.68051389 & -3.556 & 1.862 & -6.303 & -5.413 & 1.857 & 1.951 & -0.094 \\
\hline 40 & $\mathrm{II}(\mathrm{CT}-\mathrm{GD}) 1$ & 11.42675278 & 48.201 & 52.465 & -5.349 & -4.459 & 52.660 & 52.553 & -0.107 \\
\hline 41 & II(HN-AB)20 & 1076118056 & -3.513 & 2.378 & -6.757 & -5.867 & 2.354 & 2.467 & -0.113 \\
\hline 42 & II(NK-PT)13 & 21.97164722 & 4.673 & 33.962 & -30.352 & -29.462 & 34.135 & 34.019 & 0.116 \\
\hline 43 & II(AS-KS)16 & 18.46468889 & -1.303 & 22.189 & -24.336 & -23.446 & 22.143 & 22.258 & -0.115 \\
\hline 44 & II(CT-GD)4 & 11.40379444 & 33.941 & 38.783 & -5.938 & -5.048 & 38.989 & 38.870 & 0.119 \\
\hline 45 & II(DK-TM)29 & 16.10151667 & 879.636 & 890.872 & -12.078 & -11.188 & 890.824 & 890.948 & -0.124 \\
\hline 46 & II(BS-CD)14 & 10.61151389 & 56.233 & 66.101 & -10.971 & -10.081 & 66.314 & 66.190 & 0.124 \\
\hline 47 & II(HN-MT)15 & 10.36229722 & -3.939 & 1.917 & -6.711 & -5.821 & 1.882 & 2.007 & -0.125 \\
\hline 48 & $\mathrm{II}(\mathrm{AB}-\mathrm{CL}) 5$ & 10.49364444 & -2.689 & 1.950 & -5.49 & -4.600 & 1.911 & 2.039 & -0.128 \\
\hline 49 & II(BMT-DT)4 & 12.55249167 & 478.197 & 476.952 & 0.142 & 1.032 & 477.165 & 477.037 & 0.128 \\
\hline 50 & II(BMT-DT)12 & 12.29612222 & 533.210 & 531.369 & 1.000 & 1.890 & 531.320 & 531.455 & -0.135 \\
\hline 51 & II(AS-KS)32 & 18.02910833 & 35.211 & 56.839 & -22.45 & -21.560 & 56.771 & 56.909 & -0.138 \\
\hline 52 & II(TX-TL)14 & 10.45033611 & -0.452 & 2.073 & -3.363 & -2.473 & 2.021 & 2.162 & -0.141 \\
\hline 53 & II(BN-QT)11-1 & 21.05710833 & -20.878 & 4.024 & -25.996 & -25.106 & 4.228 & 4.085 & 0.143 \\
\hline 54 & $\mathrm{II}(\mathrm{HN}-\mathrm{AB}) 17$ & 10.82149722 & -4.137 & 2.018 & -6.989 & -6.099 & 1.962 & 2.107 & -0.145 \\
\hline 55 & II(TX-TL)20-1 & 1.035688889 & -2.072 & 0.536 & -3.436 & -2.546 & 0.474 & 0.626 & -0.152 \\
\hline 56 & II(TL-TV)7 & 10.08086667 & -2.189 & 0.676 & -3.693 & -2.803 & 0.614 & 0.766 & -0.152 \\
\hline 57 & II(SC-PL)29 & 9.179216667 & -3.873 & 0.910 & -5.61 & -4.720 & 0.847 & 1.002 & -0.155 \\
\hline 58 & II(NB-HN)32-1 & 21.82753333 & 67.814 & 95.958 & -29.247 & -28.357 & 96.171 & 96.016 & 0.155 \\
\hline 59 & II(AS-KS)22 & 18.28292500 & -4.080 & 18.603 & -23.488 & -22.598 & 18.518 & 18.673 & -0.155 \\
\hline 60 & $\mathrm{II}(\mathrm{CD}-\mathrm{VC}) 4$ & 10.58606111 & -5.751 & 3.242 & -9.816 & -8.926 & 3.175 & 3.331 & -0.156 \\
\hline 61 & II(HN-AB)7 & 10.83185833 & -2.929 & 4.780 & -8.531 & -7.641 & 4.712 & 4.869 & -0.157 \\
\hline 62 & II(TL-TV)5-1 & 10.16486667 & -1.941 & 1.160 & -3.922 & -3.032 & 1.091 & 1.249 & -0.158 \\
\hline 63 & II(YB-CN)24-1 & 21.20561667 & 104.775 & 133.237 & -29.254 & -28.364 & 133.139 & 133.297 & -0.158 \\
\hline 64 & II(DK-TM)45 & 15.78938333 & 386.309 & 395.440 & -9.939 & -9.049 & 395.358 & 395.517 & -0.159 \\
\hline 65 & II(AS-KS)10 & 18.61304167 & 2.407 & 26.358 & -24.749 & -23.859 & 26.266 & 26.427 & -0.161 \\
\hline 66 & II(MT-TV)11 & 9.91455000 & -0.697 & 2.012 & -3.526 & -2.636 & 1.939 & 2.103 & -0.164 \\
\hline 67 & II(DL-PR)31 & 11.66631944 & 28.014 & 23.052 & 4.153 & 5.043 & 22.971 & 23.139 & -0.168 \\
\hline
\end{tabular}


Nghiên cúu

\begin{tabular}{|c|c|c|c|c|c|c|c|c|c|}
\hline 68 & II(MX-DC)34 & 18.91195278 & -8.535 & 15.843 & -25.165 & -24.275 & 15.740 & 15.911 & -0.171 \\
\hline 69 & II(MT-TH)25 & 13.07545833 & 96.108 & 94.217 & 1.088 & 1.978 & 94.130 & 94.301 & -0.171 \\
\hline 70 & II(MT-TH)7 & 13.50634722 & 168.428 & 169.725 & -2.099 & -1.209 & 169.637 & 169.808 & -0.171 \\
\hline 71 & II(TT-KT)29 & 14.37313056 & 595.734 & 599.352 & -4.413 & -3.523 & 599.257 & 599.433 & -0.176 \\
\hline 72 & II(BMT-DT)14 & 12.21406111 & 497.959 & 495.941 & 1.223 & 2.113 & 495.846 & 496.027 & -0.181 \\
\hline 73 & II(PLK-PL)24 & 13.88302500 & 21.614 & 22.348 & -1.519 & -0.629 & 22.243 & 22.430 & -0.187 \\
\hline 74 & II(NK-PT)6-1 & 22.17745000 & 16.614 & 46.259 & -30.404 & -29.514 & 46.128 & 46.316 & -0.188 \\
\hline 75 & II(AS-KS)35 & 17.94723889 & 55.874 & 77.365 & -22.26 & -21.370 & 77.244 & 77.436 & -0.192 \\
\hline & & & & & & & Tổng $\left[\Delta_{i}^{2}\right]$ & & 0.9769 \\
\hline
\end{tabular}

Đánh giá độ chính xác:

Coi dãy trị đo là trị đo kép của cùng một đại lượng, chúng ta đánh giá độ chính xác theo công thức:

$$
m= \pm \sqrt{\frac{\left[\Delta_{i}^{2}\right]}{2 \times n}}= \pm 0.0807(\mathrm{~m})
$$

Bảng 2: Các điểm hạng I nhà nước được chọn làm các điểm cơ sở

\begin{tabular}{|c|c|c|c|c|}
\hline Tên điểm & $\begin{array}{c}\text { Các toạ độ trắc địa } \mathrm{B}, \mathrm{L} \\
\text { trong ITRF05 tương } \\
\text { ứng với ellipsoid } \\
\text { WGS84 quốc tế } \\
(0)\end{array}$ & $\begin{array}{c}\text { Độ cao trắc địa } \overline{\mathbb{B}_{z}} \text { tương ứng } \\
\text { với ellipsoid WGS84 quốc tế } \\
\text { trong hệ triều } 0 \\
(\dot{m})\end{array}$ & $\begin{array}{c}\text { Dị thường độ cao } S_{x}^{\frac{a}{s}} \\
\text { tương ứng với mặt quasi- } \\
\text { geoid khởi đầu trong hệ } \\
\text { triều } 0 \\
(\mathrm{~m})\end{array}$ & $\begin{array}{l}\text { Độ cao chuẩn } H_{z}^{\gamma} \\
\text { tương ứng với mặt } \\
\text { quasigeoid khởi đầu } \\
\text { trong hêe triều } 0 \\
(\dot{m})\end{array}$ \\
\hline $\mathrm{I}(\mathrm{HN}-\mathrm{VL}) 6-1$ & $\begin{array}{c}20.72451389 \\
105.90901390\end{array}$ & -23.625 & -26.685 & 3.066 \\
\hline $\mathrm{I}(\mathrm{HN}-\mathrm{VL}) 28-1$ & $\begin{array}{c}19.77584667 \\
105.77616190\end{array}$ & -21.895 & -24.195 & 2.291 \\
\hline $\mathrm{I}(\mathrm{HN}-\mathrm{VL}) 64$ & $\begin{array}{c}18.23650806 \\
106.02223280\end{array}$ & -19.430 & -21.801 & 2.377 \\
\hline $\mathrm{I}(\mathrm{HN}-\mathrm{VL}) 72$ & $\begin{array}{l}18.04888389 \\
106.3399558\end{array}$ & -12.452 & -20.718 & 8.265 \\
\hline I(VL-HT)98 & $\begin{array}{l}14.56539056 \\
109.0485231\end{array}$ & -1.714 & -2.724 & 1.001 \\
\hline I(VL-HT)158 & $\begin{array}{l}12.39941278 \\
109.1715708\end{array}$ & 7.578 & -4.287 & 3.291 \\
\hline I(BH-HN)33 & $\begin{array}{c}21.30917222 \\
105.40523060\end{array}$ & -15.210 & -28.321 & 13.102 \\
\hline I(BH-TH)65 & $\begin{array}{l}21.74700250 \\
103.3856356\end{array}$ & 311.806 & -31.408 & 343.222 \\
\hline $\mathrm{I}(\mathrm{BH}-\mathrm{TH}) 122 \mathrm{~A}$ & $\begin{array}{c}20.69831417 \\
105.07752670\end{array}$ & 212.958 & -26.886 & 239.838 \\
\hline
\end{tabular}


Bảng 3: Xác định độ cao chuẩn của 06 điểm

Bảng 3.1: Xác định độ cao chuẩn điểm: II(CT-VT)1

\begin{tabular}{|c|c|c|c|c|c|c|}
\hline STT & Điểm i & $\left(H_{i}^{\gamma}\right)_{z}$ & $\left\langle\bar{H}_{i j}\right)_{z}$ & $\left(\Delta \hat{\bar{\zeta}}_{i j}\right)_{z}$ & $\left(H_{j}^{\gamma}\right)_{z}$ & $\begin{array}{c}\text { Độ lệch } \\
(\mathrm{m})\end{array}$ \\
\hline 1 & $\mathrm{I}(\mathrm{HN}-\mathrm{VL}) 6-1$ & 3.066 & 20.028 & 21.692 & 1.402 & 0.008 \\
\hline 2 & $\mathrm{I}(\mathrm{HN}-\mathrm{VL}) 28-1$ & 2.291 & 18.298 & 19.202 & 1.387 & -0.007 \\
\hline 3 & $\mathrm{I}(\mathrm{HN}-\mathrm{VL}) 64$ & 2.377 & 15.833 & 16.808 & 1.402 & 0.008 \\
\hline 4 & $\mathrm{I}(\mathrm{HN}-\mathrm{VL}) 72$ & 8.265 & 8.855 & 15.725 & 1.395 & 0.001 \\
\hline 5 & $\mathrm{I}(\mathrm{VL}-\mathrm{HT}) 98$ & 1.001 & -1.883 & -2.269 & 1.387 & -0.007 \\
\hline 6 & $\mathrm{I}(\mathrm{VL}-\mathrm{HT}) 158$ & 3.291 & -11.175 & -9.280 & 1.396 & 0.002 \\
\hline 7 & $\mathrm{I}(\mathrm{BH}-\mathrm{HN}) 33$ & 13.102 & 11.613 & 23.328 & 1.387 & -0.007 \\
\hline 8 & $\mathrm{I}(\mathrm{BH}-\mathrm{TH}) 65$ & 343.222 & -315.403 & 26.415 & 1.404 & 0.010 \\
\hline 9 & $\mathrm{I}(\mathrm{BH}-\mathrm{TH}) 122 \mathrm{~A}$ & 239.838 & -216.555 & 21.893 & 1.390 & -0.004 \\
\hline & \multicolumn{7}{|c|}{ Độ cao trung bình } & 1.394 & \\
\hline
\end{tabular}

Sai số trung phương của độ cao chuẩn so với các điểm hạng I cơ sở:

$$
m_{\bar{H}^{r}}= \pm \sqrt{\frac{0.000394}{8 x 9}}= \pm 0.002 \mathrm{~m}
$$

Bảng 3.2: Xác định độ cao chuẩn điểm: II(GD-APD)2-1

\begin{tabular}{|c|c|c|c|c|c|c|}
\hline STT & Điểm i & $\left(H_{i}^{\gamma}\right)_{z}$ & $\left\langle\Delta \bar{H}_{i j}\right)_{z}$ & $\left(\Delta \hat{\bar{\zeta}}_{i j}\right)_{z}$ & $\left(H_{j}^{\gamma}\right)_{z}$ & $\begin{array}{c}\text { Độ lệch } \\
(\mathrm{m})\end{array}$ \\
\hline 1 & $\mathrm{I}(\mathrm{HN}-\mathrm{VL}) 6-1$ & 3.066 & 26.312 & 21.640 & 7.738 & 0.008 \\
\hline 2 & $\mathrm{I}(\mathrm{HN}-\mathrm{VL}) 28-1$ & 2.291 & 24.582 & 19.150 & 7.723 & -0.007 \\
\hline 3 & $\mathrm{I}(\mathrm{HN}-\mathrm{VL}) 64$ & 2.377 & 22.117 & 16.756 & 7.738 & 0.008 \\
\hline 4 & $\mathrm{I}(\mathrm{HN}-\mathrm{VL}) 72$ & 8.265 & 15.139 & 15.673 & 7.731 & 0.001 \\
\hline 5 & $\mathrm{I}(\mathrm{VL}-\mathrm{HT}) 98$ & 1.001 & 4.401 & -2.321 & 7.723 & -0.007 \\
\hline 6 & $\mathrm{I}(\mathrm{VL}-\mathrm{HT}) 158$ & 3.291 & -4.891 & -9.332 & 7.732 & 0.002 \\
\hline 7 & $\mathrm{I}(\mathrm{BH}-\mathrm{HN}) 33$ & 13.102 & 17.897 & 23.276 & 7.723 & -0.007 \\
\hline 8 & $\mathrm{I}(\mathrm{BH}-\mathrm{TH}) 65$ & 343.222 & -309.119 & 26.363 & 7.740 & 0.010 \\
\hline 9 & $\mathrm{I}(\mathrm{BH}-\mathrm{TH}) 122 \mathrm{~A}$ & 239.838 & -210.271 & 21.841 & 7.726 & -0.004 \\
\hline & \multicolumn{7}{|c|}{ Độ cao trung bình } & 7.730 & \\
\hline
\end{tabular}

Sai số trung phương của độ cao chuẩn so với các điểm hạng I cơ sở:

$$
m_{\bar{H}^{\gamma}}= \pm \sqrt{\frac{0.000394}{8 x 9}}= \pm 0.002 \mathrm{~m}
$$


Bảng 3.3: Xác định độ cao chuẩn điểm: $\|(P L K-P L) 2$

\begin{tabular}{|c|c|c|c|c|c|c|}
\hline STT & Điểm i & $\left(H_{i}^{\gamma}\right)_{z}$ & $\left(\Delta \bar{H}_{i j}\right)_{z}$ & $\left(\Delta \hat{\bar{\zeta}}_{i j}\right)_{z}$ & $\left(H_{j}^{\gamma}\right)_{z}$ & $\begin{array}{c}\text { Độ lệch } \\
(\mathrm{m})\end{array}$ \\
\hline 1 & $\mathrm{I}(\mathrm{HN}-\mathrm{VL}) 6-1$ & 3.066 & 760.424 & 23.964 & 739.526 & 0.008 \\
\hline 2 & $\mathrm{I}(\mathrm{HN}-\mathrm{VL}) 28-1$ & 2.291 & 758.694 & 21.474 & 739.511 & -0.007 \\
\hline 3 & $\mathrm{I}(\mathrm{HN}-\mathrm{VL}) 64$ & 2.377 & 756.229 & 19.080 & 739.526 & 0.008 \\
\hline 4 & $\mathrm{I}(\mathrm{HN}-\mathrm{VL}) 72$ & 8.265 & 749.251 & 17.997 & 739.519 & 0.001 \\
\hline 5 & $\mathrm{I}(\mathrm{VL}-\mathrm{HT}) 98$ & 1.001 & 738.513 & 0.003 & 739.511 & -0.007 \\
\hline 6 & $\mathrm{I}(\mathrm{VL}-\mathrm{HT}) 158$ & 3.291 & 729.221 & -7.008 & 739.520 & 0.002 \\
\hline 7 & $\mathrm{I}(\mathrm{BH}-\mathrm{HN}) 33$ & 13.102 & 752.009 & 25.600 & 739.511 & -0.007 \\
\hline 8 & $\mathrm{I}(\mathrm{BH}-\mathrm{TH}) 65$ & 343.222 & 424.993 & 28.687 & 739.528 & 0.010 \\
\hline 9 & $\mathrm{I}(\mathrm{BH}-\mathrm{TH}) 122 \mathrm{~A}$ & 239.838 & 523.841 & 24.165 & 739.514 & -0.004 \\
\hline & \multicolumn{7}{|c|}{ Độ cao trung bình } & 739.518 & \\
\hline
\end{tabular}

Sai số trung phương của độ cao chuẩn so với các điểm hạng I cơ sở:

$$
m_{\bar{H}^{\gamma}}= \pm \sqrt{\frac{0.000394}{8 x 9}}= \pm 0.002 \mathrm{~m}
$$

Bảng 3.4: Xác định độ cao chuẩn điểm: II(DK-TM)41

\begin{tabular}{|c|c|c|c|c|c|c|}
\hline STT & Điểm i & $\left(H_{i}^{\gamma}\right)_{z}$ & $\left\langle\Delta \bar{H}_{i j}\right)_{z}$ & $\left(\Delta \hat{\bar{\zeta}}_{i j}\right)_{z}$ & $\left(H_{j}^{\gamma}\right)_{z}$ & $\begin{array}{c}\text { Độ lệch } \\
(\mathrm{m})\end{array}$ \\
\hline 1 & $\mathrm{I}(\mathrm{HN}-\mathrm{VL}) 6-1$ & 3.066 & 365.355 & 17.250 & 351.171 & 0.008 \\
\hline 2 & $\mathrm{I}(\mathrm{HN}-\mathrm{VL}) 28-1$ & 2.291 & 363.625 & 14.760 & 351.156 & -0.007 \\
\hline 3 & $\mathrm{I}(\mathrm{HN}-\mathrm{VL}) 64$ & 2.377 & 361.160 & 12.366 & 351.171 & 0.008 \\
\hline 4 & $\mathrm{I}(\mathrm{HN}-\mathrm{VL}) 72$ & 8.265 & 354.182 & 11.283 & 351.164 & 0.001 \\
\hline 5 & $\mathrm{I}(\mathrm{VL}-\mathrm{HT}) 98$ & 1.001 & 343.444 & -6.711 & 351.156 & -0.007 \\
\hline 6 & $\mathrm{I}(\mathrm{VL}-\mathrm{HT}) 158$ & 3.291 & 334.152 & -13.722 & 351.165 & 0.002 \\
\hline 7 & $\mathrm{I}(\mathrm{BH}-\mathrm{HN}) 33$ & 13.102 & 356.940 & 18.886 & 351.156 & -0.007 \\
\hline 8 & $\mathrm{I}(\mathrm{BH}-\mathrm{TH}) 65$ & 343.222 & 29.924 & 21.973 & 351.173 & 0.010 \\
\hline 9 & $\mathrm{I}(\mathrm{BH}-\mathrm{TH}) 122 \mathrm{~A}$ & 239.838 & 128.772 & 17.451 & 351.159 & -0.004 \\
\hline & \multicolumn{7}{c}{ Độ cao trung bình } & 351.163 & \\
\hline
\end{tabular}

Sai số trung phương của độ cao chuẩn so với các điểm hạng I cơ sở :

$$
m_{\bar{H}^{\gamma}}= \pm \sqrt{\frac{0.000394}{8 x 9}}= \pm 0.002 \mathrm{~m}
$$


Bảng 3.5: Xác định độ cao chuẩn điểm: II(AS-KS)35

\begin{tabular}{|c|c|c|c|c|c|c|}
\hline STT & Điểm i & $\left(H_{i}^{\gamma}\right)_{z}$ & $\left(\Delta \bar{H}_{i j}\right)_{z}$ & $\left(\Delta \hat{\bar{\zeta}}_{i j}\right)_{z}$ & $\left(H_{j}^{\gamma}\right)_{z}$ & $\begin{array}{c}\text { Độ lệch } \\
(\mathrm{m})\end{array}$ \\
\hline 1 & $\mathrm{I}(\mathrm{HN}-\mathrm{VL}) 6-1$ & 3.066 & 79.499 & 5.315 & 77.250 & 0.008 \\
\hline 2 & $\mathrm{I}(\mathrm{HN}-\mathrm{VL}) 28-1$ & 2.291 & 77.769 & 2.825 & 77.235 & -0.007 \\
\hline 3 & $\mathrm{I}(\mathrm{HN}-\mathrm{VL}) 64$ & 2.377 & 75.304 & 0.431 & 77.250 & 0.008 \\
\hline 4 & $\mathrm{I}(\mathrm{HN}-\mathrm{VL}) 72$ & 8.265 & 68.326 & -0.652 & 77.243 & 0.001 \\
\hline 5 & $\mathrm{I}(\mathrm{VL}-\mathrm{HT}) 98$ & 1.001 & 57.588 & -18.646 & 77.235 & -0.007 \\
\hline 6 & $\mathrm{I}(\mathrm{VL}-\mathrm{HT}) 158$ & 3.291 & 48.296 & -25.657 & 77.244 & 0.002 \\
\hline 7 & $\mathrm{I}(\mathrm{BH}-\mathrm{HN}) 33$ & 13.102 & 71.084 & 6.951 & 77.235 & -0.007 \\
\hline 8 & $\mathrm{I}(\mathrm{BH}-\mathrm{TH}) 65$ & 343.222 & -255.932 & 10.038 & 77.252 & 0.010 \\
\hline 9 & $\mathrm{I}(\mathrm{BH}-\mathrm{TH}) 122 \mathrm{~A}$ & 239.838 & -157.084 & 5.516 & 77.238 & -0.004 \\
\hline
\end{tabular}

Sai số trung phương của độ cao chuẩn so với các điểm hạng I cơ sở:

$$
m_{\bar{H}^{r}}= \pm \sqrt{\frac{0.000394}{8 x 9}}= \pm 0.002 \mathrm{~m}
$$

Bảng 3.6: Xác định độ cao chuẩn điểm: II(LC-TG)19A

\begin{tabular}{|c|c|c|c|c|c|c|}
\hline STT & Điểm i & $\left(H_{i}^{\gamma}\right)_{z}$ & $\left(\Delta \bar{H}_{i j}\right)_{z}$ & $\left(\Delta \hat{\bar{\zeta}}_{i j}\right)_{z}$ & $\left(H_{j}^{\gamma}\right)_{z}$ & $\begin{array}{l}\text { Độ lệch } \\
\text { (m) }\end{array}$ \\
\hline 1 & I(HN-VL)6-1 & 3.066 & 485.744 & -5.353 & 494.163 & 0.008 \\
\hline 2 & $\mathrm{I}(\mathrm{HN}-\mathrm{VL}) 28-1$ & 2.291 & 484.014 & -7.843 & 494.148 & -0.007 \\
\hline 3 & $\mathrm{I}(\mathrm{HN}-\mathrm{VL}) 64$ & 2.377 & 481.549 & -10.237 & 494.163 & 0.008 \\
\hline 4 & $\mathrm{I}(\mathrm{HN}-\mathrm{VL}) 72$ & 8.265 & 474.571 & -11.320 & 494.156 & 0.001 \\
\hline 5 & I(VL-HT)98 & 1.001 & 463.833 & -29.314 & 494.148 & -0.007 \\
\hline 6 & $\mathrm{I}(\mathrm{VL}-\mathrm{HT}) 158$ & 3.291 & 454.541 & -36.325 & 494.157 & 0.002 \\
\hline 7 & $\mathrm{I}(\mathrm{BH}-\mathrm{HN}) 33$ & 13.102 & 477.329 & -3.717 & 494.148 & -0.007 \\
\hline 8 & $\mathrm{I}(\mathrm{BH}-\mathrm{TH}) 65$ & 343.222 & 150.313 & -0.630 & 494.165 & 0.010 \\
\hline 9 & $\mathrm{I}(\mathrm{BH}-\mathrm{TH}) 122 \mathrm{~A}$ & 239.838 & 249.161 & -5.152 & 494.151 & -0.004 \\
\hline \multicolumn{5}{|c|}{ Độ cao trung bình } & 494.156 & \\
\hline
\end{tabular}

Sai số trung phương của độ cao chuẩn so với các điểm hạng I cơ sở:

$$
m_{\bar{H}^{r}}= \pm \sqrt{\frac{0.000394}{8 x 9}}= \pm 0.002 \mathrm{~m}
$$

Nhận xét: So sánh kết quả xác định độ cao chuẩn của 06 điểm hạng II từ 09 điểm hạng I cơ sở được thống kê trong bảng 3.1, 3.2, 3.3, 3.4, 3.5, 3.6 ta thấy, sai số trung phương của độ cao chuẩn so với các điểm hạng I cơ sở chỉ ở mức $\pm 2 \mathrm{~mm}$.

\section{Kết luận}

Qua kết quả kiểm tra đánh giá độ chính xác của mô hình quasigeoid quốc gia khởi đầu 
VIGAC2014 bằng cách xác định độ cao chuẩn của các điểm độ cao hạng II thông qua hai phương pháp khác nhau, ta đi đến các kết luận sau:

- Mô hình VIGAC2014 có độ chính xác có thể đáp ứng được công tác xác định độ cao chuẩn tương đương hạng II. Tuy nhiên, để sử dụng mô hình này cần đo đạc lại và xử lý toán học mạng lưới độ cao hạng I, II quốc gia do nhiều mốc độ cao quốc gia đã bị xê dịch trên thực địa dước các tác nhân tự nhiên và nhân sinh;

- Mô hình VIGAC2014 có độ chính xác đồng đều trên lãnh thổ Việt Nam;

- Trong tương lai, tiếp tục nâng cao độ chính xác của mô hình VIGAC2014 (cao hơn \pm $4 \mathrm{~cm}$ ) dựa vào việc hiệu chỉnh các hệ số điều hòa cầu của mô hình EGM2008 cho phù hợp với địa hình lãnh thổ Việt Nam từ dứ liệu trọng lực ở Việt Nam.O

\section{Tài liệu tham khảo}

[1]. Bursa M., Kenyon S., Kouba J., Radj K., Vatrt V., Vojtiskov., Simek J. (2002). World Height System specified by geopotential at tide stations. IAG Symposia 124, 291 - 296. Springer.

[2]. Dennis D. McCarthy and Gerard Petit (2004).IERS Conventions (2003). IERS Technical Note No. 32. Frankfurt am Main, 2004.

[3]. Hà Minh Hòa (2003). Nghiên cứu xác định độ chính xác cho phép của độ cao chuẩn các hạng và giải quyết một số vấn đề liên quan đến việc xây dựng mô hình Kvazigeoid chính xác nhờ hệ tọa độ động học. Tạp chí KHKT Mỏ - Địa chất, số 3, tháng 7/2003, trg. $110-114$.

[4]. Hà Minh Hòa (2007). Giải quyết một số vấn đề liên quan đến việc chuyển hệ độ cao được xác định từ mặt nước biển trung bình ở trạm nghiệm triều về mặt quasigeoid toàn cầu. Tạp chí Địa chính số 2 , tháng 4/2007, trg. 3 - 11.

[5]. Hà Minh Hòa (2012a). Nghiên cứu xác định thế năng trọng trường thực $W_{0}$ của mặt Geoid cục bộ trùng với mặt biển trung bình tại trạm nghiệm triều Hòn Dấu. Tuyễn tập báo cáo hội nghị Khoa học và Công nghệ. Trắc địa và Bản đồ vì sự nghiệp Tài nguyên và Môi trường. Hà Nội - tháng 10/2012, trg. 10 - 16.

[6]. Hà Minh Hòa (2012b). Nghiên cứu cơ sở khoa học của việc hoàn thiện hệ độ cao gắn liền với việc xây dựng hệ tọa độ động lực quốc gia. Báo cáo tổng kết khoa học và kỹ thuật của đề tài nghiên cứu khoa học cấp Bộ. Bộ Tài Nguyên và Môi Trường. Hà Nội $12 / 2012$.

[7]. Hà Minh Hòa (2012c). Nghiên cứu xác định thế năng trọng trường thực $\mathrm{W}_{0}$ của mặt Geoid cục bộ trùng với mặt biển trung bình tại trạm nghiệm triều Hòn Dấu. Tuyễn tập báo cáo hội nghị Khoa học và Công nghệ. Trắc địa và Bản đồ vì sự nghiệp Tài nguyên và Môi trường. Tháng 10/2012, trg. 10 - 16.

[8]. Hà Minh Hòa (2014). Lý thuyết và thực tiễn của trọng lực trắc địa. NXB Khoa học và Kỹ thuật, 590 trg. Hà Nội 2014.

[9]. Hà Minh Hòa (2015a). Nghiên cứu hoàn thiện phương pháp xử lý toán học mạng lưới độ cao hạng I, II quốc gia trong hệ độ cao hiện đại ở Việt Nam. Báo cáo tổng kết khoa học và kỹ thuật. Đề tài cấp Bộ tài nguyên và Môi trường, Hà Nội - tháng 8/2015. 
[10]. Hà Minh Hòa, Nguyễn Thị Thanh Hương, Lương Thanh Thạch (2015b). Đánh giá kiểm tra thế trọng trường $\mathrm{W}_{0}$ của mặt geoid cục bộ Hòn Dấu trên cơ sở sử dụng 89 điểm độ cao hạng I. Tạp chí Khoa học Đo đạc và Bản đồ. Số 26 - 12/2015, trg. 1- 5.

[11]. Hà Minh Hòa và nnk (2016). Nghiên cứu đánh giá các mặt chuẩn mực nước biển (mặt "0" độ sâu, trung bình và cao nhất) theo các phương pháp trắc địa, hải văn và kiến tạo hiện đại phục vụ xây dựng các công trình và quy hoạch đới bờ Việt Nam trong xu thế biến đổi khí hậu. Báo cáo tổng hợp kết quả khoa học công nghệ đề tài. Chương trình khoa học và công nghệ trọng điểm cấp Nhà nước mã số : KC.09/11-15, Bộ Khoa học và Công nghệ, năm 2016;

[12]. International Hydrographic Organization (IHO), (2011). Resolutions of the International Hydrographic Organization, 2nd edition, Publication M-3, 2010, updated to July 2012. Monaco: IHO.

[13]. Nadim DAYOUB (Syria), Philip MOORE, Stuart J. EDWARDS and Nigel T. PENNA (TheUnited Kingdom) (2011).The Geoid Geopotential Value for Unification of Vertical Datums. FIG Working Week 2011. Bridging the Gap between Cultures. Marrakech, Morocco, 18-22 May 2011.

[14]. Nguyễn Tuấn Anh (2015). Nghiên cứu chi tiết độ cao của mặt Geoid cục bộ Hoàn Dấu so với mặt Geoid toàn cầu trên lãnh thổ Việt Nam. Tạp chí Khoa học Đo đạc và Bản đồ. Số 25 - 9/2015, $\operatorname{trg} .33$ - 38.

[15]. Petit G., Luzum B.(2010). IERS Conventions (2010). IERS Technical Note No. 3 , Verlag des Bundesamts fur Kartographie und Geodasie. Frankfurt am Main 2010, 179pp.

[16]. Smirnov N.V., Belugin D. A. (1969). Lý thuyết xác suất và toán học thống kê trong ứng dụng trắc địa. Matxcơva, Nedra, 1969, 379 trg. (Tiếng Nga).

[17]. TenzerR.,Vatrt V., Amos M (2009). Realization of the World Height System in New Zealand: Preliminary Study, pp 343-349, Volume 136 of the series International Association of Geodesy Symposia. Geodesy for Planet Earth.

[18]. Vũ Xuân Cường (2015). Hiện tượng lún mốc độ cao quốc gia tại khu vực phía Nam và giải pháp khắc phục. Tạp chí Khoa học Đo đạc và Bản đồ. Số 24 - 6/ 2015, trg. 30 - 3;

[19].wgs84@nga.mil(EGM2008 - WGS 84 Version).O

\section{Summary}

Accuracy assessment of the starting VIGAC2014 national Quasigeoid model based on 75 elevation points of second-order national level

\section{Luong Thanh Thach, Ha Noi University of Natural Resources and Environment}

This study was carried out to test to assess thefield gravity valueand the elevation value $W_{0}=62636847.291 \mathrm{~m}^{2} \cdot \mathrm{s}^{-2}$ of the local quasigeoid at Hon Dau $\mathrm{H}_{0}=0.890 \mathrm{~m}$ withthe purpose for assessing the accuracy of the starting VIGAC2014 national Quasigeoid model based on 75 elevation points of second-order national level. The test results confirmed that the reliability of these above values to set the basis ofscience to solve the problem of modern geophysical survey in Vietnam. $O$ 